\title{
Proposta e aplicação de um instrumento de acompanhamento da implantação do SW-CMM Nível 2
}

\author{
Sabrina Marczak, Jorge Audy, Luciana Sá \\ Pontifícia Universidade Católica do Rio Grande do Sul - PUCRS \\ Faculdade de Informática - Grupo QUATES (QUAlidade e TEste de Software) ${ }^{*}$ \\ Av. Ipiranga, 6681 - Prédio 30, Bloco IV - Porto Alegre, RS - CEP 90619-900 \\ \{smarczak; audy\}@inf.pucrs.br, salucian@yahoo.com
}

\begin{abstract}
The Capability Maturity Model for Software has been used by many organizations as a way to improve its software processes. The aim of this paper is to present an instrument (questionnaire) developed to track the $S W$ CMM Level 2 implantation process and to describe an experience about its application. The instrument developed and a critical analyses of the results obtained by the instrument application are presented as contribution.
\end{abstract}

Resumo. O Capability Maturity Model for Software tem sido utilizado por muitas organizações como ferramenta para melhorar seus processos de software. O objetivo deste artigo é propor um instrumento (questionário) de acompanhamento da implantação do $S W$-CMM Nivel 2 e apresentar o relato da experiência de acompanhamento desta implantação em uma organização (das duas onde já foi aplicado). Como contribuição, apresenta-se o instrumento desenvolvido e uma análise crítica dos resultados obtidos.

\section{Introdução}

Buscando aumentar a sua produtividade, reduzir os custos e melhorar a qualidade e confiabilidade de seus produtos, muitas empresas têm distribuído o desenvolvimento de seus projetos de software para terceiros. Neste contexto, um dos modelos bastante adotado é o SW-CMM. Para que modelos como este sejam implantados com sucesso, é vital que as organizações planejem e acompanhem o processo de implantação, bem como os recursos e os investimentos.

Uma equipe de pesquisa de uma grande universidade particular do sul do país vem acompanhando a implantação deste modelo de qualidade nas organizações instaladas no parque tecnológico desta universidade. Estabeleceu-se um processo de acompanhamento da implantação do Nível 2 do CMM (o qual não é foco deste artigo) e vem-se validando o mesmo durante as atividades com as empresas. Uma das formas de se acompanhar a evolução do conhecimento dos colaboradores das empresas foi através da aplicação de um questionário elaborado pelos pesquisadores.

Desta forma, o objetivo deste artigo é propor um instrumento (questionário) de acompanhamento da implantação do SW-CMM Nível 2 e apresentar o relato da experiência de acompanhamento desta implantação em uma organização de desenvolvimento de software offshore de uma multinacional da área de TI. Além de apresentar o instrumento em si, descreve-se sua inserção no processo de implantação

\footnotetext{
* Para maiores informações sobre este grupo de pesquisa, consulte http://www.inf.pucrs.br/ quates
} 
dos processos de desenvolvimento de software da organização mencionada, tendo por objetivo o acompanhamento da evolução da apropriação dos conceitos teóricos envolvidos, como subsídio complementar às avaliações intermediárias e oficiais ao longo do processo de avaliação. Finalmente, é feita uma análise crítica dos resultados obtidos com a aplicação do instrumento de pesquisa.

O artigo está organizado em cinco seções. A Seção 2 descreve o método de pesquisa utilizado. O processo de definição e implantação do SW-CMM Nível 2 na organização estudada e as aplicações do instrumento durante este processo, bem como as ações tomadas como resultado da análise dos resultados identificados são apresentadas na Seção 3. A Seção 4 apresenta a análise crítica dos resultados obtidos e das aplicações do instrumento e a Seção 5 apresenta as referências bibliográficas. No Apêndice A, apresenta-se o instrumento proposto.

\section{Método de pesquisa}

Esta pesquisa se caracteriza como um estudo predominantemente exploratório, o qual tem como principal finalidade desenvolver, esclarecer e modificar conceitos e idéias, com vistas à formulação de novas teorias, modelos e hipóteses pesquisáveis em estudos posteriores [1]. O método de pesquisa foi o estudo de caso, adotado conforme proposto por Yin [2]. O estudo de caso foi desenvolvido em uma organização de desenvolvimento de software offshore de uma organização americana durante o processo de implantação do SW-CMM Nível 2. Um ano depois (2004) foi aplicado, com as devidas adequações, no processo de implantação do CMMI Nível 2 de uma empresa européia que possui uma operação de desenvolvimento de software offshore no Brasil. Esta foi reconhecida como uma organização Nível 2 em fevereiro de 2005.

Este relato de experiência apresenta o processo original desenvolvido na primeira organização. A mesma contava com 120 colaboradores no momento da realização da pesquisa trabalhando em projetos que atendem as necessidades da área de TI da empresa. Neste estudo foram identificadas as dificuldades da organização em relação à implantação do SW-CMM Nível 2 e soluções (ações e práticas) para colocar em prática os processos definidos para atender as necessidades da organização, bem como para se atingir com sucesso a avaliação formal destes processos. Utilizou-se como principal instrumento de pesquisa um questionário, elaborado pela equipe de pesquisa, para acompanhar o processo de implantação do SW-CMM Nível 2 na organização, medindo a evolução dos conhecimentos adquiridos pelos colaboradores ao longo do processo. O questionário contém 50 questões, de diferentes naturezas: objetivas, perceptivas e descritivas. Estas questões abrangeram os seguintes constructos teóricos: Processo de Desenvolvimento de Software; Qualidade de Software; Cultura Organizacional e Áreas-chave do SW-CMM Nível 2. A Tabela 1 apresenta a organização do questionário.

Inicialmente, definiu-se os constructos e a natureza das questões e, logo após, realizou-se uma série de reuniões para definição das questões, bem como à forma como estas seriam distribuídas. Realizou-se refinamentos sucessivos até chegar-se a uma versão do questionário disponibilizada para validação. Foram realizadas validações de face e de conteúdo [3] por dois especialistas da área de Sistemas de Informação. A atividade seguinte foi a realização do pré-teste, onde se aplicou o questionário em três colaboradores da empresa, sendo um da área de desenvolvimento de software e dois da 
área de qualidade de software. Com esta aplicação pode-se identificar inconvenientes, eliminar equívocos e ambigüidades, e estimar o tempo necessário para resposta do questionário. Baseado nas considerações e sugestões indicadas por ambos os envolvidos no processo de validação do questionário, elaborou-se uma nova versão do questionário, validada pela direção da empresa. Tendo feitos alguns ajustes, chegou-se então a versão final do questionário, apresentado no Apêndice A. Cabe ressaltar que algumas alterações foram feitas para possibilitar sua divulgação.

Tabela 1 - Organização do questionário

\begin{tabular}{c|c|c|c|c}
\hline \multirow{2}{*}{ Tipo de questão } & \multicolumn{4}{|c}{ Constructos } \\
\cline { 2 - 5 } & $\begin{array}{c}\text { Processo de Desenv. } \\
\text { de Software }\end{array}$ & $\begin{array}{c}\text { Qualidade de } \\
\text { Software }\end{array}$ & $\begin{array}{c}\text { Cultura } \\
\text { Organizacional }\end{array}$ & $\begin{array}{c}\text { Áreas-chave do SW- } \\
\text { CMM Nível 2 }\end{array}$ \\
\hline Objetivas & 2 & 4 & 4 & 3 \\
\hline Perceptivas & 2 & 2 & 11 & 10 \\
\hline Descritivas & 7 & 1 & 4 & 0 \\
\hline Total & 11 & 7 & 19 & 13 \\
\hline
\end{tabular}

Tendo definido o questionário, este foi aplicado em dois momentos distintos do processo de implantação do SW-CMM Nível 2. A primeira aplicação aconteceu cinco meses antes da avaliação formal e a segunda, há menos de um mês desta avaliação. $\mathrm{O}$ total de respondentes na primeira aplicação foi de 74 , de uma população de 90 colaboradores, e na segunda aplicação foram 98 respondentes de uma população de 120 pessoas. O público respondente envolveu todos os colaboradores da empresa, desde o nível diretivo até o nível operacional. A análise dos dados foi realizada com uso de um software de tabulação e análise de dados, denominado Sphinx, e contou com o apoio de uma especialista no uso da ferramenta e na análise de dados. Após a análise dos resultados da primeira aplicação, a equipe de pesquisa propôs um conjunto de ações e atividades para endereçar as dificuldades identificadas e assim, atingir as expectativas quanto à avaliação formal. Além de elaborar as propostas apresentadas, parte da equipe participou ativamente da realização das atividades, podendo vivenciar o impacto das ações no dia-a-dia dos colaboradores e a evolução do conhecimento destes.

\section{Aplicação do instrumento de acompanhamento da implantação do Nível 2}

\subsection{Processo de definição e implantação do SW-CMM Nível 2}

A primeira organização iniciou o processo de definição e implantação do SW-CMM Nível 2 em março de 2001 e o concluiu em janeiro de 2003, após a avaliação e reconhecimento como uma organização SW-CMM nível 2 de maturidade. Este processo de definição e implantação do SW-CMM Nível 2 foi organizado em fases, conforme descrito a seguir.

A Fase I (março a agosto/2001) caracterizou-se principalmente pela contratação de profissionais habilitados para conduzir o processo de implantação do SW-CMM e pelos treinamentos sobre o modelo. A Fase II (abril a setembro/2001) ocorreu em paralelo com a anterior e iniciou com a realização de um diagnóstico para identificar o nível de maturidade do processo de desenvolvimento de software da organização. A Fase III (outubro/2001 a novembro/2002) é considerada a fase de estabilização dos 
processos, na qual os mesmos foram disponibilizados para utilização dos colaboradores e melhorados conforme os retornos recebidos. A partir deste momento a equipe de pesquisa passou a acompanhar as atividades realizadas no contexto do processo de implantação, registrando e analisando suas execuções com o objetivo de fornecer uma visão externa e isenta do real andamento do processo. Durante a Fase IV (novembro/2002 a janeiro/2003) foram realizadas várias avaliações intermediárias em preparação para a avaliação oficial, em janeiro de 2003. Os resultados destas avaliações foram usados para corrigir as falhas identificadas e melhorar alguns aspectos já definidos. A Fase $V$ (última semana de janeiro/2003) envolveu a avaliação oficial da organização nas áreas-chave do Nível 2 do SW-CMM. Nas cinco áreas-chave do Nível 2 avaliadas foi encontrado grau zero de não conformidade, sendo a empresa reconhecida como uma organização Nível 2 de maturidade.

\subsection{Acompanhamento da implantação do SW-CMM Nível 2}

O acompanhamento da implantação do SW-CMM Nível 2 pela equipe de pesquisa não se restringiu às aplicações do questionário e o plano de ações proposto. A equipe também participou de um conjunto de atividades, atuando segundo diferentes papéis previstos nos processos definidos (por exemplo, como auditor de qualidade e membro da equipe de avaliação), o que viabilizou o acompanhamento do dia-a-dia das atividades da organização e uma melhor compreensão do processo de implantação do Nível 2.

Após ter passado um mês elaborando e validando o questionário, a equipe planejou sua aplicação em três momentos: antes do primeiro treinamento oficial que seria ministrado sobre os processos, buscando identificar o conhecimento prévio dos colaboradores; dois meses após o primeiro treinamento, para identificar a evolução do conhecimento e os resultados práticos da institucionalização dos processos; e um mês às vésperas da avaliação oficial, para identificar se os colaboradores estavam sentindo-se aptos a serem consultados durante a avaliação. Em função de algumas mudanças estratégicas em relação às atividades planejadas para a institucionalização dos processos, aplicou-se o questionário somente duas vezes.

A primeira aplicação do questionário ocorreu durante a Fase III. Sob orientação dos gerentes, todos os colaboradores da organização suspenderam suas atividades durante um turno de trabalho (quatro horas). A equipe de pesquisa forneceu as instruções de preenchimento do questionário e, ao final de quase duas horas, todos os colaboradores o haviam respondido e a equipe já estava planejando a tabulação e análise dos dados. Tabulados os dados, organizou-se diferentes estratos de análise e gerou-se os gráficos com os resultados. De posse dos gráficos, a equipe discutiu os vários resultados encontrados e elaborou um relatório apresentando os principais resultados dos diferentes estratos e explanando os mesmos.

Em uma reunião informal, que concentrou quase a totalidade dos colaboradores da organização, foram apresentados os resultados obtidos. Os colaboradores tiveram a oportunidade de discutir suas dúvidas sobre o processo de implantação do SW-CMM e até mesmo sobre a importância estratégica da avaliação formal para a organização. Segundo relatos, foi nesta ocasião que muitos dos colaboradores sentiram-se, pela primeira vez, membros participantes do processo de definição e implantação dos processos do Nível 2, pois nem todos tinham participado dos grupos de definição dos processos. Também, foi neste momento que se sentiram motivados a colaborar e 
trabalhar na busca do reconhecimento oficial de uma organização Nível 2 de maturidade SW-CMM. A equipe de pesquisa elaborou um conjunto de ações e atividades buscando contribuir para melhorar aqueles aspectos falhos do processo.

As ações foram organizadas abordando diferentes aspectos e agrupadas da seguinte forma: Plano de capacitação e reforço de conteúdos; Formalização do Plano Estratégico (nível conceitual) da organização; e Elaboração de um plano de comunicação formal. Parte destas ações foram desenvolvidas em conjunto ou exclusivamente pelos pesquisadores. O plano de capacitação e reforço de conteúdos foi organizado conforme os processos a serem utilizados e baseado nos papéis exercidos pelos colaboradores, e alguns aspectos culturais que precisavam ser discutidos, levandose em consideração que a organização trabalha em constante contato com a matriz. Teve-se dois meses de treinamento intensivo sobre os processos e os assuntos relacionados. Organizou-se diversos mini-cursos e palestras com um professor convidado, de uma universidade americana, especialista na influência de aspectos culturais no desenvolvimento de software.

Quanto à formalização do Plano Estratégico da organização, o coordenador da equipe de pesquisa (doutor na área de Planejamento Estratégico de Sistemas de Informação) auxiliou o diretor na formalização da missão, visão e políticas da organização. Todos os colaboradores foram consultados e tiveram oportunidade de opinar. Diversas reuniões foram organizadas até chegar-se na versão adotada pela organização. $O$ treinamento sobre o plano estratégico da organização foi organizado e conduzido pela equipe junto a uma psicóloga, convidada a participar para realizar atividades em grupo e envolver os participantes em atividades motivadoras. $\mathrm{O}$ plano de comunicação formal foi organizado exclusivamente pela equipe de pesquisa, a qual responsabilizou-se pela elaboração de cartazes motivacionais e informativos, adesivos, concursos internos, jogos e performances teatrais, todos relacionados à implantação dos processos e buscando desinibir os colaboradores a falarem sobre sua nova forma de trabalho após a institucionalização dos processos. Por ter participado ativamente no grupo de atividades relacionadas aos mecanismos de comunicação formais da organização, a equipe pode identificar o progresso do grupo, bem como as mais diversas formas de reação sobre as atividades realizadas, permitindo uma avaliação das atividades planejadas.

A segunda aplicação do questionário ocorreu no início da Fase $V$, às vésperas da avaliação formal. A aplicação aconteceu sob as mesmas circunstâncias anteriores, sob coordenação da equipe de pesquisa. O tempo de resposta nesta segunda aplicação reduziu-se quase à metade do anterior, levando um pouco mais de uma hora. Os colaboradores sentiram-se mais à vontade para responder às questões, tanto por já estarem mais familiarizados com o assunto, como por já saberem o objetivo da aplicação, que não se caracterizou como avaliativa de performance e sim da evolução do conhecimento adquirido no decorrer da institucionalização dos processos definidos pela organização. Os dados foram tabulados e analisados conforme a primeira aplicação do questionário e, novamente, as descobertas da pesquisa foram entregues aos gestores da organização na forma de um relatório consolidado. 


\section{Análise crítica dos resultados}

Os resultados obtidos nesta pesquisa sustentam, conforme dados apresentados, a importância de acompanhar-se o processo de implantação em modelos de melhoria do processo de software para que se possa tomar medidas corretivas no andamento do processo. Relatou-se o acompanhamento em uma organização específica baseado em um instrumento de pesquisa. Este foi desenvolvido com o objetivo de identificar a evolução do conhecimento dos colaboradores da organização em relação aos processos sendo implantados e ao modelo de referência em si. O questionário e o processo descrito foram utilizados também no processo de avaliação CMMI Nível 2 uma grande empresa européia de desenvolvimento de software, com igual sucesso.

$\mathrm{O}$ instrumento de pesquisa (questionário) mostrou-se útil para o sucesso do processo de implantação do modelo de qualidade nas duas organização estudadas. Além de permitir a identificação de carências significativas em conhecimentos específicos nas áreas-chave do Nível 2 do SW-CMM e do CMMI, também permitiu atuar de forma rápida em outras carências identificadas. Como exemplo, destaca-se os aspectos relacionados à capacitação dos colaboradores, tanto em relação à gerência de projetos como desenvolvimento e teste de software; à comunicação interna e definição dos referenciais estratégicos da organização.

Ao comparar os resultados identificados após as sistemáticas aplicações do questionário, foi possível perceber uma evolução no conhecimento sobre os processos de desenvolvimento de software definidos, nas áreas-chave pertinentes e até mesmo sobre a importância das avaliações informais e formais, em função dos referenciais estratégicos da organização, desenvolvidos nas duas empresas no contexto da aplicação do instrumento proposto. Atribui-se parte desta evolução ao plano de ações proposto e colocado em prática, em sua maioria, pela equipe de pesquisa, tendo por base os resultados obtidos nas aplicações sistemáticas do instrumento de pesquisa proposto no processo de implantação.

\section{Referências bibliográficas}

[1] GIL, A. Métodos e técnicas de pesquisa social. São Paulo: Atlas, 1995.

[2] YIN, R. Estudo de Caso: Planejamento e Métodos. Porto Alegre: Bookman, 2001.

[3] HOPPEN, N. Evaluation research of article in Information Systems: Proposal of one guide. XXI ANPAD Proceedings: Rio de Janeiro, 1997.

\section{APÊNDICE A: QUESTIONÁRIO PARA ACOMPANHAMENTO DA IMPLANTAÇÃo DO SW-CMM NÍVEL 2}

\section{ObJetivo Deste QuestionáRio}

Este questionário faz parte de um estudo de um Centro de Pesquisa que acompanha e estuda o processo de implantação do SW-CMM Nível 2 em uma organização. O objetivo desta pesquisa é identificar o grau de entendimento dos colaboradores da organização referente ao processo de implantação do modelo de melhoria de software na organização. Destaca-se que este questionário não possui caráter avaliativo pontual, busca apenas acompanhar a evolução dos conhecimentos adquiridos ao longo do processo de implantação do SW-CMM. O propósito é tirar uma "foto" do momento atual do processo. Neste contexto, estão previstas três aplicações deste questionário. Salienta-se que as respostas individuais serão de conhecimento unicamente do grupo de pesquisa. Somente serão apresentados à organização ou publicados os resultados gerais consolidados. 


\section{Simpósio Brasileiro de Qualidade de Software}

\section{INSTRUÇÕES GERAIS}

Responda ao questionário apenas com base nos seus conhecimentos, sem consultar fontes de apoio.

\section{ESTRUTURA Do QUESTIONÁRIO}

O questionário é composto de questões que abrangem as áreas de processo de software, qualidade e cultura organizacional. Há três tipos de questões: i) Objetivas; ii) Perceptivas; e iii) Descritivas.

\section{DADOS DEMOGRÁFICOS}

\section{Identificação Pessoal}

Nome: Idade:

Anos

Escolaridade (informe somente o maior grau)
$\square 1^{\circ} \mathrm{Grau}$
$2^{\circ} \mathrm{Grau}$
Superior Incompleto
Superior Completo
Especialização
$\square$ Mestrado / MBA Incompleto
Mestrado / MBA Completo

Identifique as informações solicitadas abaixo conforme a escolaridade assinalada acima

Curso:

Universidade:
Ano de conclusão:

Anos

Tempo de experiência profissional na área de Informática:

Tempo de empresa

Até 6 meses

$\square$ De 7 a 12 meses

Contratado

Vínculo:

Equipe:

Equipe 1
De 1 a 2 ano

Terceirizado
De 2 a 5 anos

Estagiário

Função: Equipe 2 Outra:

(Conforme a terminologia utilizada na organização)

\section{QUESTÕES OBJETIVAS}

Instruções de Preenchimento: nas questões de 01 a 09 você deve assinalar apenas uma das alternativas fornecidas; na questão 10 você deve relacionar a coluna da direita com a coluna da esquerda; na questão 11 , ordenar as alternativas apresentadas de acordo com a sua opinião, e nas questões 12 e 13 você deve assinalar uma ou mais das alternativas fornecidas.

1. Qual das metodologias abaixo é adotada como base para o processo de desenvolvimento da organização?
a) Modelagem de Dados
b) Rational Unified Process (RUP) c) Análise Estruturada
d) Extreme Programming (XP)
e) Microsoft Solutions Framework (MSF)

2. Qual o modelo de ciclo de vida utilizado pela metodologia adotada como base para o processo de desenvolvimento de software da organização?
a) Incremental e cascata
b) Espiral
d) Prototipação
e) Incremental
c) Espiral e cascata

3. O que é o framework de processos?
a) Ferramenta de teste
b) Repositório de arquivos
c) Ferramenta de comunicação sobre o status dos projetos
d) Conjunto de todos os processos, procedimentos e templates
e) Nome da Intranet da organização

4. O framework de processos é resultado da combinação entre as contribuições de quais modelos?
a) MSF e SPICE
b) $C M M$ e $M S F$
c) ISO 12207 e CMM
d) CMM e RUP
e) ISO 12207 e RUP

5. Qual o principal fator que tem comprometido o uso efetivo do framework de processos?

a) Pressão por prazos de entrega dos projetos

b) Falta de conhecimento do conteúdo

c) Falta de apoio gerencial

d) Discordância quanto ao que está definido

e) Incompatibilidade com a forma de trabalho do cliente

6. Qual das opções abaixo apresenta somente áreas-chaves relacionadas ao nível de maturidade que a organização está buscando atingir neste momento?

a) Defect Prevention, Software Configuration Management e Training Program 


\section{Simpósio Brasileiro de Qualidade de Software}

b) Software Quality Assurance, Peer Reviews e Organization Process Definition

c) Requirements Management, Software Project Tracking and Oversight $e$ Software Configuration Management

d) Software Quality Assurance, Software Project Planning e Defect Prevention

e) Software Subcontract Management, Technology Change Management e Software Project Tracking and Oversight

7. Estabelecer e manter a integridade dos produtos de software ao longo do ciclo de vida do projeto é responsabilidade de qual das seguintes áreas-chave do modelo CMM?
a) Software Quality Assurance
b) Software Product Engineering
c) Organization Process Definition
d) Technology Change Management
e) Software Configuration Management

8. De quem é a responsabilidade de autorizar mudanças na baseline de configuração de software?

a) Software Quality Assurance (SQA)

b) Infrastructure Group (Logistics)

c) Program Manager (PM)

d) Software Configuration Control Board (SCCB)

e) Software Engineering Process Group (SEPG)

9. Preencha a coluna da direita de acordo com o respectivo nível de maturidade do CMM indicado na coluna da esquerda.

a) Definido b) Otimizado c) Gerenciado d) Repetível e) Inicial

Nível 1 (e) Nível 2(d) Nível 3(a) Nível 4 (c) Nível 5 (b)

10. Qual dos gráficos abaixo melhor representa a transição de uma tecnologia antiga (TA) para uma nova (TN), ao longo do tempo?
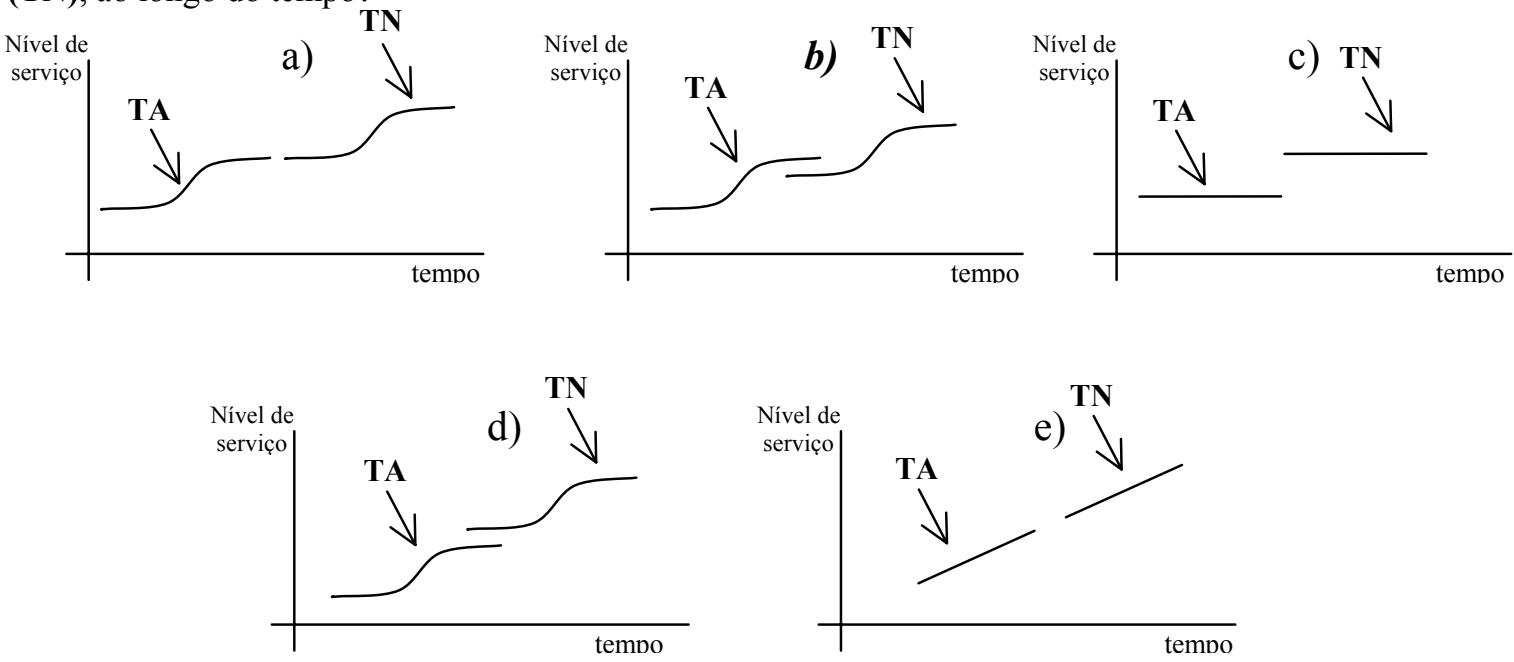

11. Classifique quem, na sua opinião, mais tem apoiado a organização na busca pela certificação CMM. (Numerar de 1 - menos tem apoiado a 5 - mais tem apoiado)
( 5 ) Diretor da organização
( 2 ) Setor de TI da filial brasileira
( 1 ) SEPG da matriz
(4) Chefia imediata
( 3 ) SEPG da organização

12. Qual(is) artefato(s) abaixo é(são) tipicamente gerado(s) durante a fase de planejamento (Planning phase) de um projeto? (Assinale uma ou mais alternativas)
( ) Source Code
( ) Test Plan
( ) Change Request
( ) Vision/Scope
( ) Technical Specification (Design)

13. Qual(is) o(s) principal(is) fator(es) que tem dificultado o seu envolvimento nas atividades dos grupos de trabalho de definição dos processos (PAT's)? (Assinale uma ou mais alternativas)

( ) Indisponibilidade de tempo ( ) Desinteresse pessoal (desmotivação) ( ) Falta de apoio gerencial

( ) Desconhecimento do que está ocorrendo ( ) Atividade não relacionada com a minha função

\section{QUESTÕES PERCEPTIVAS}

Instruções de Preenchimento: as questões abaixo abordam aspectos práticos e teóricos, relevantes no contexto desta pesquisa. Você deve classificar o seu grau de percepção das acertivas de acordo com a escala fornecida. O nível de concordância abrange os valores de 1 a 5 , sendo o valor 1 relacionado a "Discordo Totalmente" e o 5 a "Concordo Totalmente". Caso desconheça, marque "Desconheço".

14. O CMM parte do princípio que mesmo sem um processo definido os produtos são de qualidade.

15. A forma como estão disponibilizadas as informações no framework de processos é prática e eficiente. 


\section{Simpósio Brasileiro de Qualidade de Software}

16. A documentação formal dos processos é imprescindível para tornar repetível o processo de desenvolvimento.

\begin{tabular}{c|c|c|c|c|c|l|c|}
$\begin{array}{c}\text { Discordo } \\
\text { totalmente }\end{array}$ & $\square$ & $\square$ & $\square$ & $\square$ & $\square$ & $\begin{array}{l}\text { Concordo } \\
\text { totalmente }\end{array}$ & $\square$ \\
\cline { 2 - 7 } & $\mathbf{1}$ & $\mathbf{2}$ & $\mathbf{3}$ & $\mathbf{4}$ & $\mathbf{5}$ & Desconheço
\end{tabular}

17. Todos os projetos que você participa atualmente na organização têm seus requisitos documentados, conforme estabelecido no framework de processos.

18. Todas as mudanças de requisitos são documentadas e gerenciadas nos projetos em que você participa

19. Em todos os projetos de software que você participa atualmente na organização, os requisitos especificados foram utilizados como base para o seu planejamento.

20. Todos os projetos que você participa atualmente na organização seguem um plano documentado, conforme definido no framework de processos.

21. Para todos os projetos de software que você está participando na organização são feitas estimativas do tamanho dos produtos, estimativas de esforço das atividades e cronograma.

22. Revisões de acompanhamento de projeto são realizadas periodicamente em todos os projetos que você participa atualmente na organização.

23. Relatórios de status do projeto são produzidos semanalmente em todos os projetos que você participa.

24. Os problemas surgidos no decorrer dos projetos em desenvolvimento na organização são gerenciados adequadamente, conforme proposto no framework de processos.

25. O treinamento recebido sobre of framework de processos foi suficiente.

26. Os compromissos assumidos com grupos de fora da equipe de projeto não precisam ser registrados no Plano do Projeto

27. As atividades do SQA contribuem efetivamente para o sucesso do projeto.

28. O SQA tem como principal responsabilidade testar os produtos gerados pelos projetos.

29. A responsabilidade pela definição dos processos de software é exclusiva do SEPG.

30. O SEPG tem criado condições de todos os colaboradores da organização apresentarem contribuições ao processo de implantação do CMM.

31. Existe uma clara vinculação entre o trabalho do SEPG e as práticas atuais nos projetos em andamento. 32. A direção da organização foi clara ao apresentar as políticas de desenvolvimento de software.

33. O estilo gerencial dos gestores (diretor e gerentes seniores) da organização é participativo, envolvendo o pessoal nos diversos níveis de tomada de decisão.

34. O estilo gerencial dos gerentes de projeto é participativo, envolvendo o pessoal na tomada de decisão. 35. A implantação do processo de melhoria de software tem impactado negativamente (excesso de formalismo, pressão por prazos, etc.) nas minhas atividades.

36. A divulgação das informações quanto ao andamento dos trabalhos relacionados à implantação do processo de melhoria de software (etapas, próximas fases, resultados, etc.) é suficiente.

37. Os mecanismos de comunicação internos da organização, relativos aos projetos em desenvolvimento e atividades em geral, é transparente e eficaz.

38. O esforço da organização em buscar uma melhoria no processo de desenvolvimento de software está gerando uma redução no nível de estresse e de pressão no ambiente de trabalho.

\section{QUESTÕES DESCRITIVAS}

Instruções de Preenchimento: responda as questões abaixo de acordo com seu conhecimento.

39. Quais as fases da metodologia adotada na organização como base para o desenvolvimento?

40. Segundo o framework de processos, quais são os tipos de planos gerados na fase de planejamento que todos os projetos devem elaborar?

41. No instanciamento de um novo projeto de desenvolvimento de software na organização, quais são os papéis típicos que compõem a equipe interna de projeto?

42. Diferencie Software product de Software work product.

43. O que é uma baseline do ponto de vista do processo de Gerência de Configuração de Software?

44. Descreva, com suas palavras, o que você entende por qualidade de software.

45. No processo de auditoria de qualidade que o SQA realiza, quais são os padrões e referências utilizados para confrontar o que está sendo realizado com o que foi planejado?

46. Como deve proceder o SQA ao identificar uma não-conformidade?

47. Qual é a missão da organização?

48. Quais são as principais políticas de desenvolvimento de software da organização?

49. Quais as principais ações estratégicas da organização?

50. Quem são os clientes da organização? 